brevis to the pelvis, the ramus of the pubes and the borders of the thyroid foramen being distinctly felt from the inside of the sac. All the wall of the cyst which could be dissected from the surrounding parts was now cut away, and what remained firmly adherent to the muscles was rubbed with caustic potash. The wound was then plugged with lint, and some water. dressing applied. During the operation, which lasted about three-quarters of an hour, but little blood was lost. A few ligatures were applied to some small spouting vessels. The fluid evacuated during the operation contained several small, soft, and light-coloured masses of fungus. Portions of the sac were blended with muscular fibre, in character tough and leathery, and principally composed of cellular tissue. In the evening, the wound was dressed. The inferior portion was brought together by sutures and isinglass plaster, superiorly being left open. Water-dressing and bandage. Very restless all night. Headache and vomiting, with heavy expression about the eyes. Ordered, twenty-five minims of laudanum.

6th.-This morning, he was weak, oppressed, and restless; pulse 160 , very weak and small; surface warm. Brandy-andegg mixture was given as a stimulant. Wound looking healthy, but with considerable serous discharge. At noon, he became much worse; the radial pulse imperceptible, while the respiration is deep and sighing; considerable thirst and pallor, with very much uneasiness and restlessness. The bandage was withdrawn, and the wound opened up; no hæmorrhage was detected. Mr. Erichsen saw him in the afternoon, and ordered several of the sutures to be removed, and a large poultice to envelope the whole of the upper and inner aspect of the thigh. To be kept up with beef-tea, brandy, and wine; and to have effervescing salines, to allay thirst and nausea. In the evening, he rallied a little, and the rudial pulse was again felt.

7th. - No sleep last night, though he had a draught of morphia; constant exhibition of stimulants during the night; considerable lividity of surface of face, lips, and extremities; quite pulseless; sighing respiration; no cardiac murmur nor complication; lungs healthy, no dulness of bases; surface cold; face swollen; frontal and occipital headache; no affection of vision. Urine scanty, of most offensive odour, turbid, greenish-brown, with considerable deposit of the same colour; notable quantity of albumen. Under the microscope were seen an enormous quantity of renal epithelium, much granular matter and débris, and many casts of large diameter. In the afternoon the lividity considerably increased, and the boy sank and died at half-past four P.M.

Post-mortem Examination, forty hours after death.-Heart healthy; over the surface of the left ventricle and auricle a number of dark, purple, hæmorrhagic patches, closely resembling those of purpura hæmorrhagica. Blood generally of the body rather fluid, and unhealthy looking, dark in colour. Left kidney large, pale, and flabby, with little distinction between cortical and pyramidal substances; appears to be in the early stage of grannlar degeneration; weight, six ounces and a half. Right kidney smaller, and more healthy looking; weight, four ounces; capsules separate readily in both. All the other viscera healthy. On dissecting the limb, it was found that the cyst lay between the adductor magnus behind, and the adductors longus and brevis and the pectineus in front. Superiorly it extended up in front of the quadratus lumborum and the obturator externus. What remained of the cyst was firmly incorporated and joined with the adductors magnus and brevis; the substance of the former is very much indurated, and the cellular tissue amongst the fibres very much hypertrophied.

\section{CHARING-CROSS HOSPITAL.}

HYSTERICAL PAIN IN THE ABDOMEN.

(Under the care of Mr. Canton.)

Perhaps no author has contributed more to the advancement of our knowledge in the recognition of those peculiar manifestations of hysteria which occasionally present them selves to the notice of the surgeon than Sir Benjamin Brodie; and though little has been added to our capability of compre hending the intricate nature of this Protean disease, we have severtheless gained much in an extended acquaintance both with its forms and frequency.

In the accompanying case, the hysterical symptoms were well-marked generally and locally, so as to leave no doubt of the nature of the complaint, as little, indeed, as in the numerous instances which are to be met with of simulated spinal or knee-joint disease, more especially in the higher walks of 7ife. The morbid sensibility of the skin, when the hand is even gently applied, or, as it were, merely glancing over it, is a 372 very characteristic feature; and the unconsciousness of pain, when the attention is diverted and firm pressure still maintained on its site, is also a notable peculiarity in these affections. In addition, we remark here other symptoms which so commonly bespeak the presence of hysteria, or, more properly speaking, which accompany and develope the disease-namely, uterine irregularity, characterized by fits, a peculiar form of crying, copious and limpid urine, constipated bowels and flatulence, and a sensation of choking produced by the wellknown globus hystericus. Difficulty of micturition was also present, which not unfrequently amounts to actual retention. With respect to this latter symptom, we may refer to the lectures of Dr. Tyler Smith, now publishing in this Journal (see The Lancex, Jan. 12th, p. 29): "In hysterical females, the clitoris is sometimes subject to a constant erection, almost similar to that which obtains in priapism in the male. During this condition, it is difficult or impossible to pass urine-a point first noticed, I believe, by Dr. Sylvester, of Clapham."

This symptom-retention of urine-is often perpetuated by that unhappy pruriency of feeling over which the patient is unable to exercise control, and which seeks a morbid gratification in the means we commonly employ for emptying the bladder.

In commenting upon this case, Mr. Canton remarked that in this hospital the sister of the ward is charged with the duty of withdrawing the urine in hysterical retention, - a circumstance which tends materially to diminish the frequency in the requirement of the performance of this operation. In illustration of the peculiar phases sometines assumed by this inscratable complaint, it may be mentioned that nearly twenty years ago a young female in the same hospital, under the care of Dr. Shearman, laboured under hysteria which very closely simulated hydrophobia; and two cases of the genuine form of the latter disease having died in the hospital only a short time previous, the medical men were enabled fully to appreciate the resemblance which the feigned disease bore to the true form.

Elizabeth L_- unmarried, aged eighteen, states that ten days previous to her application at the hospital, and whilst sitting on the sill to clean the windows, she fell back suddenly and was much frightened, but did not strike herself, nor was she unseated. She continued her work with ease until towards the evening, when pain suddenly attacked her in the right inguinal region, and opposite to the situation of the ovary. The pain is stated to be internally, as it were, in the pelvis, and is not felt at the surface. On the accession of pain she screamed out and "fell into hysterics;" in which condition she continued for upwards of an bour. When recovered, she found the pain abated, but it became augmented a short time afterwards, and has continued off and on ever since. She applied to a dispensary and rubbed soap-liniment on her side, as directed, but the pain and inconvenience were increased by it. Two or three days after her fright, difficulty of micturition became a prominent symptom, and at the same time the inconvenience in her side was greater, especially so as the urine was being voided, and for a few minutes subsequently: she finds herself much easier after urinating. The secretion is often very limpid, and passed in large quantities. Says she is very hysterical, and subject to sobbing fits, if she is unduly excited, and de. scribes the inconvenience she often feels from the sensation we denominate globus hysterius. At fourteen years of age she menstruated: the first flux was inordinate, and weakened her much; since this time the menses have always been very scanty, but the periods have been tolerably natural. The flow generally lasts for two, or it may be three days, and occasionally it appears for one only. The secretion is very pale, and has been particularly so since her fright; and at the same time the system does not seem to be so relieved by it as is usually the case. Is not troubled with any inconvenience in the mamme. The bowels are ordinarily confined, and she is much troubled with wind. Her face is remarkable from being almost completely covered with spots of acne punctata.

On first examination at the seat of inconvenience, the patient began imrnediately to cry in an hysterical manner, and as soon as the hand was even in the slightest manner applied to the surface; whilst deeper or more decided pressure, directed inwards or downwards, increased the pain, which she did not, however, complain of when her attention, at the time, was directed to some other subject. There was neither tumefaction, redness, increased temperature, or ecchymosis of the part; and manipnlation of the corresponding portion of the abdomen gave no inconvenience.

This case is undergoing treatment, but no very decided effect has as yet been produced upon the disease, although an 1 improvement in the general symptoms is manifest. 